\title{
Research on the Extraction Process in Aqueous Two-Phase System of Moringa Leaf Flavonoids
}

\author{
Rui Wang ${ }^{1,2 *}$, Xueqin Zhang ${ }^{2}$ and Qian Zhou $^{2}$ \\ ${ }^{1}$ Chongqing Collaborative Innovation Center for Functional Food, Chongqing University of Education, Chongqing, 400067, China \\ ${ }^{2}$ College of Biological and Chemical Engineering, Chongqing University of Education, Chongqing, 400067, China
}

\begin{abstract}
Moringa leaves were used as raw materials, ethanol and ammonium sulfate were used to construct a aqueous two-phase system to extract the flavonoids from Moringa leaves. The solid-liquid ratio, ethanol content, ultrasonic extraction time, and ultrasonic extraction temperature were selected as single factors, and the extraction process of flavonoids was optimized through single-factor experiments and orthogonal experiments. The optimal process was: 1:110 of solid-liquid ratio, $45 \%$ of ethanol concentration, 15 minutes of ultrasonic extraction time, $70^{\circ} \mathrm{C}$ of ultrasonic extraction temperature. Under this condition, the extraction rate of Moringa leaf flavonoids reached $8.37 \%$.
\end{abstract}

\section{Introduction}

Moringa belongs to the Moringa family. Moringa leaves contain a lot of trace elements, tweenty kinds of amino acids and a variety of antioxidants, and low fat and cholesterol content. Moringa leaf powder could supply nutrition for human, has the functions of lowering blood pressure, lowering blood lipids and blood sugar, and preventing cancer and tumors. Studies have found that Moringa leaves contain a lot of flavonoids, and the flavonoids have anti-inflammatory, antioxidant, antiaging, treatment of various acute and chronic diseases, and anti-cancer effects. They have application prospects in the fields of health care and medicine [1-3]. Extracting flavonoids by the method of aqueous two-phase system , the instruments used in the operation process are relatively simple, and the instruments and various reagents used are also cheap, so it could save money. Moreover, the conditions required for the aqueous two-phase system are mild, which could save experiment time. The most important thing is that the use of aqueous two-phase system could obtain a higher extraction rate, so the method of aqueous two-phase system is more and more applied to the fields of food and medicine research [4-5]. This study aims to explore the extraction process of Moringa leaf flavonoids by aqueous two-phase system, and the results could provide a theoretical basis application of Moringa leaf in food and medicine.

\section{Experimental method}

\subsection{The standard curve}

$20 \mathrm{mg}$ rutin was accurately weighed, then dissolved with
$30 \%$ ethanol and diluted into a $100 \mathrm{~mL}$ brown volumetric flask to prepare a standard solution of rutin with a concentration of $0.2 \mathrm{mg} / \mathrm{mL}$. The pipette was used to accurately pipette $0 \mathrm{~mL}, 1.0 \mathrm{~mL}, 2.0 \mathrm{~mL}, 3.0 \mathrm{~mL}, 4.0 \mathrm{~mL}$ and $5.0 \mathrm{~mL}$ standard solution to $25.0 \mathrm{~mL}$ colorimetric tube respectively. $0.7 \mathrm{~mL}$ of $5 \% \mathrm{NaNO}_{2}$ solution was added to the colorimetric tube. After the reaction for 6 minutes, 0.7 $\mathrm{mL}$ of $10 \% \mathrm{Al}\left(\mathrm{NO}_{3}\right)_{3}$ solution was added to the colorimetric tube. After reacting for 6 minutes, $10.0 \mathrm{~mL}$ of $1 \mathrm{~mol} / \mathrm{L} \mathrm{NaOH}$ solution was added to the colorimetric tube, then $30 \%$ ethanol was added to make the volume, mixed thoroughly, and measured the absorbance after reacting for 15 minutes. The detection wavelength was $510 \mathrm{~nm}$. The absorbance value was set as the ordinate (Y) and the concentration of the standard solution was set as the abscissa $(\mathrm{X})$. The standard curve was drawn and the standard curve equation was obtained [6].

\subsection{Aqueous two-phase extraction of Moringa leaf flavonoids}

2.2.1 Preparation of ethanol-ammonium sulfate aqueous two-phase system. The dosage of ammonium sulfate was fixed and dissolved with a certain amount of deionized water, and the dosage of absolute ethanol was gradually changed. when the Moringa leaf powder was added to the solution, the solution could appear up and down layering and could remain stable. After that, the aqueous two-phase system was successfully established [7-12].

2.2.2 Extraction of flavoniods. $0.2 \mathrm{~g}$ of Moringa leaf powder was added in an Erlenmeyer flask according to the method of preparing an aqueous two-phase system, $3.0 \mathrm{~g}$ of anhydrous ammonium sulfate and $10.0 \mathrm{~mL}$ deionized

*Corresponding author's e-mail: wangrui@cque.edu.cn 
water were added, shaken well. Then $10.0 \mathrm{~mL}$ of absolute ethanol was added, shaken well and standed still. After the solution had obvious upper and lower stratification, it indicated that the ethanol-ammonium sulfate aqueous two-phase system was successfully established. After the established ethanol-ammonium sulfate aqueous two-phase system was treated by ultrasonic at $60^{\circ} \mathrm{C}$ for 10 minutes, the extract was put into a separatory funnel for static layering. After 10 minutes, the upper and lower phases of the extract were collected with a graduated cylinder and recorded the volumes respectively.The upper and lower two-phase extracts were put into a high-speed centrifuge, centrifuged at $3500 \mathrm{r} / \mathrm{min}$ for 10 minutes. Then, the extracts were ready for further experiment. $2.0 \mathrm{~mL}$ of the centrifuged upper layer extract was pipetted and added into a $25.0 \mathrm{~mL}$ colorimetric tube, then the content of Moringa leaf flavonoids was detected by the method of the determination of rutin standard solutions in previous.

\subsection{Single factor experiments}

2.3.1 The effect of solid-liquid ratio. $0.2 \mathrm{~g}$ of Moringa leaf powder was weighed in an Erlenmeyer flask, and the solid-liquid ratio was set as 1:100, 1:110, 1:120, 1:130, $1: 140$. Then, the effect of the solid-liquid ratio on the extraction rate of Moringa leaves was studied under the extraction temperature of $60{ }^{\circ} \mathrm{C}$, the ultrasonic time of 10 minutes, and the ethanol concentration of $50 \%$.

2.3.2 The effect of ethanol concentration. $0.2 \mathrm{~g}$ of Moringa leaf powder was weighed in an Erlenmeyer flask, and the ethanol concentration was set as $35 \%, 40 \%, 45 \%$, $50 \%$ and $55 \%$. Then, the effect of the ethanol concentration on the extraction rate of Moringa leaves was studied under the the extraction temperature of $60^{\circ} \mathrm{C}$, the ultrasonic time of 10 minutes, and the solid-liquid ratio of $1: 100$.

2.3.3 The effect of ultrasonic extraction time. $0.2 \mathrm{~g}$ of Moringa leaf powder was weighed in an Erlenmeyer flask, and the ultrasonic extraction time was set as 5 minutes, 10 $\mathrm{min}, 15 \mathrm{~min}, 20 \mathrm{~min}, 25 \mathrm{~min}$. Then, the effect of the ethanol concentration on the extraction rate of Moringa oleifera leaves was studied under the the extraction temperature of $60{ }^{\circ} \mathrm{C}$, the ethanol concentration of $50 \%$, and the solid-liquid ratio of $1: 100$.

\subsubsection{The effect of ultrasonic extraction temperature.} $0.2 \mathrm{~g}$ of Moringa leaf powder was weighed in an Erlenmeyer flask, and the ultrasonic extraction temperature was set as $40{ }^{\circ} \mathrm{C}, 50{ }^{\circ} \mathrm{C}, 60{ }^{\circ} \mathrm{C}, 70{ }^{\circ} \mathrm{C}$ and $80^{\circ} \mathrm{C}$. Then, the effect of the ethanol concentration on the extraction rate of Moringa leaves was studied under the ultrasonic time of 10 minutes, the ethanol concentration of $50 \%$ and the solid-liquid ratio of $1: 100$.

\subsection{Orthogonal experiment}

The appropriate factor levels were analyzed and selected based on the above single-factor test results. The L9 $\left(3^{4}\right)$ orthogonal test based on the solid-liquid ratio, ethanol concentration, ultrasonic extraction time, and ultrasonic extraction temperature was designed. Then, the optimal extraction process was obtained.

\section{Results}

\subsection{The standard curve}

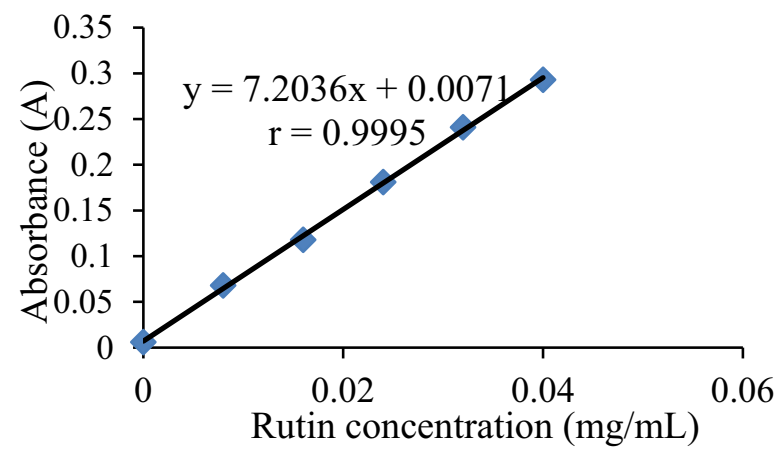

Figure 1. Rutin standard curve

As shown in Figure 1, the standard curve equation was $y=7.2036 x+0.071$, and the Correlation coefficient was suitable, and could be used in the calculation of the concentration of flavonoids.

\subsection{The effect of solid-liquid ratio on extraction rate of flavonoids}

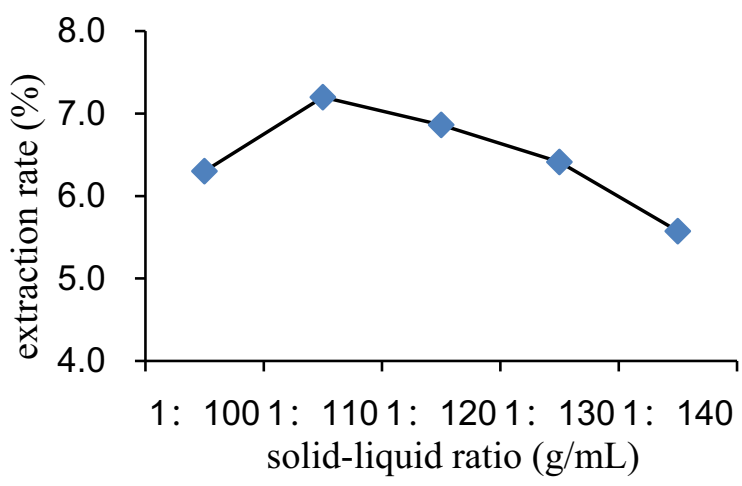

Figure 2. The effect of solid-liquid ratio on extraction rate of flavonoids

When the solid-liquid ratio was 1:110, the extraction rate of Moringa leaf flavonoids reached the maximum value (Figure 2), so the best solid-liquid ratio $(\mathrm{m} / \mathrm{v})$ used in this experiment was 1: 110 . 


\subsection{The effect of ethanol concentration on extraction rate of flavonoids}

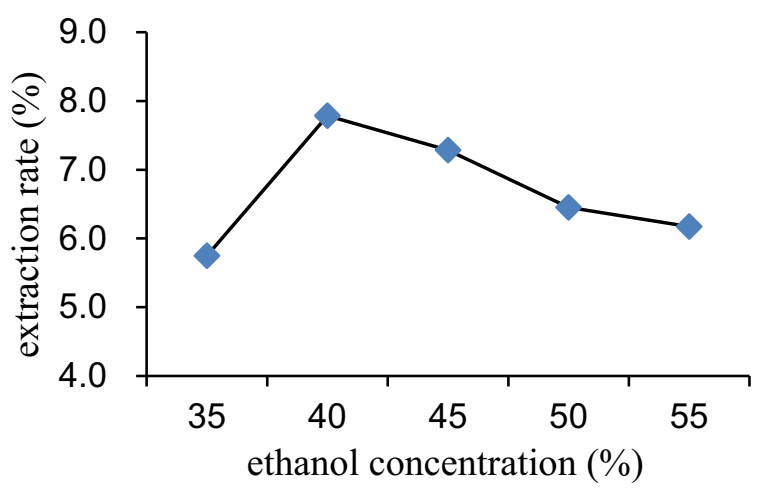

Figure 3. The effect of ethanol concentration on extraction rate of flavonoids

As could be seen from Figure 3, when the ethanol concentration was $40 \%$, the extraction rate of Moringa leaf flavonoids reached the maximum value. However, when the ethanol concentration was too high, the extraction rate decreased continuously, perhaps because anhydrous ammonium sulfate was insoluble in absolute ethanol. Therefore, $40 \%$ was the optimal ethanol concentration used in this experiment.

\subsection{The effect of ultrasonic extraction time on extraction rate of flavonoids}

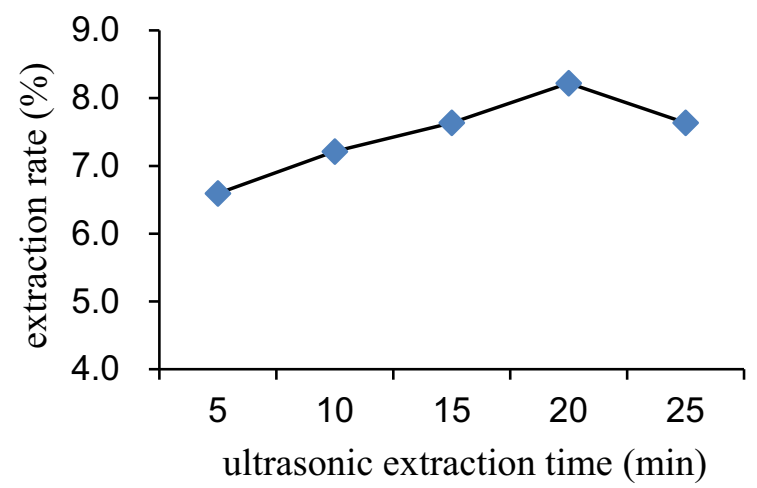

Figure 4. The effect of ultrasonic extraction time on extraction rate of flavonoids

It could be seen from Figure 4 that when the ultrasonic extraction time was 20 minutes, the extraction rate of Moringa leaf flavonoids reached the maximum value, which might be due to the accelerated evaporation of ethanol at a temperature of $60{ }^{\circ} \mathrm{C}$ for a long time, which affected the aqueous two-phase system. When the extraction time was 25 minutes, the extraction rate began to decrease.

\subsection{The effect of ultrasonic extraction temperature on extraction rate of flavonoids}

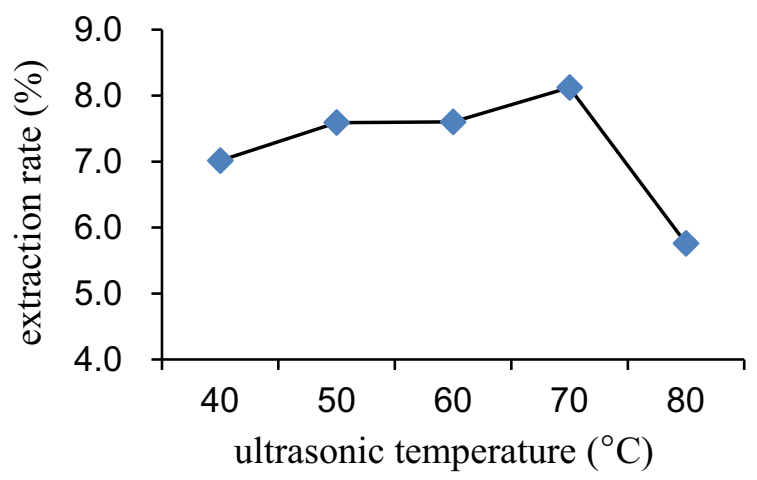

Figure 5. The effect of ultrasonic temperature on extraction rate of flavonoids

As shown in Figure 5, when the ultrasonic extraction temperature was $70^{\circ} \mathrm{C}$, the extraction rate of Moringa leaf flavonoids reached the maximum value, perhaps because the temperature was too high and the ethanol volatilized and affected the aqueous two-phase system, so the extraction rate began to descend at $80^{\circ} \mathrm{C}$. Therefore, $70^{\circ} \mathrm{C}$ was the best ultrasonic extraction temperature used in this experiment.

\subsection{The result of orthogonal experiments}

Based on the results of single factor experiments, the orthogonal experiments were carried out with solid-liquid ratio, ethanol concentration, ultrasonic extraction time and ultrasonic extraction temperature as the factors to determine the best extraction process (Table 1). The $\mathrm{R}$ value obtained from the orthogonal experiment results was analyzed (Table 2), that could be seen that the ethanol concentration was the most important factor, and the ultrasonic extraction temperature was the least important factor. Then, combined the $\mathrm{K}$ value of each factor in Table 2 , the optimal extraction scheme determined in this experiment was $\mathrm{A}_{2} \mathrm{~B}_{3} \mathrm{C}_{1} \mathrm{D}_{2}$. In order to verify whether the scheme optimized by the orthogonal experiment was the optimal, a verification experiment was performed on the scheme $\mathrm{A}_{2} \mathrm{~B}_{3} \mathrm{C}_{1} \mathrm{D}_{2}$ to determine whether the extraction rate was the maxmun value. The scheme $\mathrm{A}_{2} \mathrm{~B}_{3} \mathrm{C}_{1} \mathrm{D}_{2}$ was subjected to the extraction experiment and the extraction rate of flavonoids was $8.37 \%$, which was close to the maximum extraction rate obtained in the orthogonal experiment process of $8.45 \%$. It can be concluded that the scheme $\mathrm{A}_{2} \mathrm{~B}_{3} \mathrm{C}_{1} \mathrm{D}_{2}$ was the best extraction scheme. Therefore, the optimal process was: 1:110 of solid-liquid ratio, $45 \%$ of ethanol concentration, 15 minutes of ultrasonic extraction time, $70{ }^{\circ} \mathrm{C}$ of ultrasonic extraction temperature.

Table 1. Factors and levels of orthogonal experiments

\begin{tabular}{ccccc}
\hline & A & B & C & D \\
& solid-liquid ratio $(\mathrm{g} / \mathrm{mL})$ & ethanol concentration $(\%)$ & extraction time $(\mathrm{min})$ & $\begin{array}{c}\left.{ }^{\circ} \mathrm{C}\right) \\
\text { temperature }\left({ }^{\circ} \mathrm{C}\right)\end{array}$ \\
\hline 1 & $1: 100$ & 35 & 15 & 60 \\
2 & $1: 110$ & 40 & 20 & 70 \\
3 & $1: 120$ & 45 & 25 & 80 \\
\hline
\end{tabular}


Table 2. Results and analysis of orthogonal experiments

\begin{tabular}{|c|c|c|c|c|c|}
\hline entry & $\begin{array}{l}\text { solid-liquid ratio } \\
\qquad(\mathrm{g} / \mathrm{mL})\end{array}$ & $\begin{array}{l}\text { ethanol concentration } \\
(\%)\end{array}$ & $\begin{array}{l}\text { extraction } \\
\text { time (min) }\end{array}$ & $\begin{array}{l}\text { temperature } \\
\qquad\left({ }^{\circ} \mathrm{C}\right)\end{array}$ & extraction rate $(\%)$ \\
\hline 1 & $1: 100$ & 35 & 15 & 60 & 6.04 \\
\hline 2 & $1: 100$ & 40 & 20 & 70 & 6.51 \\
\hline 3 & $1: 100$ & 45 & 25 & 80 & 5.62 \\
\hline 4 & $1: 110$ & 35 & 20 & 80 & 5.78 \\
\hline 5 & $1: 110$ & 40 & 25 & 60 & 7.16 \\
\hline 6 & $1: 110$ & 45 & 15 & 70 & 8.45 \\
\hline 7 & $1: 120$ & 35 & 25 & 70 & 5.79 \\
\hline 8 & $1: 120$ & 40 & 15 & 80 & 7.02 \\
\hline 9 & $1: 120$ & 45 & 20 & 60 & 7.07 \\
\hline $\mathrm{K} 1$ & 181.56 & 176.12 & 211 & 202.61 & \\
\hline $\mathrm{K} 2$ & 209.91 & 206.86 & 193.55 & 203.45 & \\
\hline $\mathrm{K} 3$ & 198.77 & 207.26 & 185.69 & 184. 18 & \\
\hline $\mathrm{k} 1$ & 68.52 & 58.71 & 70.33 & 67.54 & \\
\hline $\mathrm{k} 2$ & 69.97 & 68.95 & 64.52 & 67.82 & \\
\hline $\mathrm{k} 3$ & 66.26 & 69.09 & 61.90 & 61.40 & \\
\hline $\mathrm{R}$ & 28.35 & 31.14 & 25.31 & 19.27 & \\
\hline order & & $\mathrm{B}>\mathrm{A}>\mathrm{C}>\mathrm{D}$ & & & \\
\hline $\begin{array}{l}\text { optimization } \\
\text { levels }\end{array}$ & $\mathrm{A}_{2}$ & $\mathrm{~B}_{3}$ & $\mathrm{C}_{1}$ & $\mathrm{D}_{2}$ & \\
\hline $\begin{array}{l}\text { optimization } \\
\text { condition }\end{array}$ & & $\mathrm{A}_{2} \mathrm{~B}_{3} \mathrm{C}_{1} \mathrm{D}_{2}$ & & & \\
\hline
\end{tabular}

\section{Conclusion}

In this research, a aqueous two-phase system was used to extract the flavonoids from Moringa leaves. By studying the effect of four factors such as solid-liquid ratio, ethanol concentration, ultrasonic extraction time and ultrasonic extraction temperature on the extraction rate, and combining with orthogonal experiments to optimize the extraction process, the optimal extraction scheme was determined. Through a comprehensive study of the $\mathrm{K}$ and $\mathrm{R}$ values at various factor levels, the optimal extraction scheme in this experiment was obtained as $\mathrm{A}_{2} \mathrm{~B}_{3} \mathrm{C}_{1} \mathrm{D}_{2}$, and verification experiments on this scheme showed that the extraction rate of flavonoids from Moringa leaves could reach $8.37 \%$. Therefore, the optimal process was: $1: 110$ of solid-liquid ratio, $45 \%$ of ethanol concentration, 15 minutes of ultrasonic extraction time, $70{ }^{\circ} \mathrm{C}$ of ultrasonic extraction temperature.

\section{Acknowledgments}

This research was funded by Teaching Reform Project of Chongqing University of Education (JG201730).

\section{References}

1. Zhou, Y.P., Sun, H., Yan, J.Z., Shi, S.H., Ge, Z.H. (2016) Edible method and effect of Moringa. Modern Rural Science and Technology, 22: 72.
2. Sun, M.Y., Wang, Y.M. (2007) The optimization of extraction method of total flavoids from the leaves of moringa oleifera. Journal of Shaoguan University, 12: 88-92.

3. Luo, Q.Q. (2018) The processing technology of Moringa tea. Fujian Science \& Technology of Tropical Crops, 43:39-40.

4. Zhang, H., Wang, S. (2014) Aqueous two-phase and microwave method to extract and purifytotal flavonoids in nicandra physaloids. Journal of Harbin University of Commerce (Natural Sciences Edition), 30:220-223.

5. Hao, H.Y., Niu, M.L., Wang, Y., Fan, Y.L., Sun, L.H. (2016) Extraction of Total Flavonoids from Purslane Using Ethanol-Ammonium Sulfate Aqueous Two Phase System. Journal of Anhui Agri.Sci, 44:145-147.

6. Huang, H.N., Dong, X.T. (2018) Optimization of Extraction Process of Flavonoids from Chrysanthemum morifoliumby Response Surface Methodology. Modern Agricultural Technology, 24:245-251.

7. Ma, Y., GU L.Z., Bao W.C., Wu, Z.X., Liu, Z.S. (2018) Study on aqueous two-phase extraction and antioxidant activity of anthocyanin from purple sweet potato. China Food Additives, 6:73-79.

8. Wang, F., Liao, L., Huang, Y., Zhao, X.M., Hou, J.N., Cao, W.L., Li, D., Li, X.W. (2016) Extraction of proanthocyanidins from rapeseed meal with aqueous two-phase system. Science and Technology of Food Industry, 37:303-308. 
9. Fan, Y., Lu, Y.M., Tan, C.P., Liang, Y., Cui, B. (2016) Aqueous Two-phase Extraction for Capsaicin, 41:2226.

10. Huang, Y., Duan, Y., Duan, J.H., Huang, W., Bai, Y.J., Zhang, P.L., Zheng, F., Feng, Z.S. (2016) Extraction of anthocyanin from grape skin with aqueous twophase system. Science and Technology of Food Industry, 37:220-225.

11. Hao, H.Y., Zhou, F., Wang, G.L., Gao, Y.Y., Ding, X.J. (2017) Extraction of Flavonoids from Herba houttuyniae Using Ethanol-Ammonium Sulfate Aqueous Two Phase System. Hubei Agricultural Sciences, 56:4844-4846.

12. Zhuo, L., Ruan, S.Y., Deng, H.Y. (2012) Study on the extraction of flavonoids from blood orange residue by aqueous two-phase method. Journal of Chifeng University (Natural Science Edition), 28:17-18. 